\title{
REGIONS OF VARIABILITY FOR GENERALIZED $\alpha$-CONVEX AND $\beta$-STARLIKE FUNCTIONS, AND THEIR EXTREME POINTS
}

\author{
Shaolin Chen and Aiwu Huang
}

\begin{abstract}
Suppose that $n$ is a positive integer. For any real number $\alpha$ ( $\beta$ resp.) with $\alpha<1\left(\beta>1\right.$ resp.), let $K^{\langle n\rangle}(\alpha)\left(K^{\langle n\rangle}(\beta)\right.$ resp. $)$ be the class of analytic functions in the unit disk $\mathbb{D}$ with $f(0)=f^{\prime}(0)=\cdots=$ $f^{(n-1)}(0)=f^{(n)}(0)-1=0, \operatorname{Re}\left(\frac{z f^{(n+1)}(z)}{f^{(n)}(z)}+1\right)>\alpha\left(\operatorname{Re}\left(\frac{z f^{(n+1)}(z)}{f^{(n)}(z)}+1\right)<\right.$ $\beta$ resp.) in $\mathbb{D}$, and for any $\lambda \in \overline{\mathbb{D}}$, let $K^{\langle n\rangle}(\alpha, \lambda)\left(K^{\langle n\rangle}(\beta, \lambda)\right.$ resp. $)$ denote a subclass of $K^{\langle n\rangle}(\alpha)\left(K^{\langle n\rangle}(\beta)\right.$ resp. $)$ whose elements satisfy some condition about derivatives. For any fixed $z_{0} \in \mathbb{D}$, we shall determine the two regions of variability $V^{\langle n\rangle}\left(z_{0}, \alpha\right)\left(V^{\langle n\rangle}\left(z_{0}, \beta\right)\right.$ resp. $)$ and $V^{\langle n\rangle}\left(z_{0}, \alpha, \lambda\right)$ $\left(V^{\langle n\rangle}\left(z_{0}, \beta, \lambda\right)\right.$ resp.). Also we shall determine the extreme points of the families of analytic functions which satisfy $f(\mathbb{D}) \subset V^{\langle n\rangle}\left(z_{0}, \alpha\right)(f(\mathbb{D}) \subset$ $V^{\langle n\rangle}\left(z_{0}, \beta\right)$ resp.) when $f$ ranges over the classes $K^{\langle n\rangle}(\alpha)\left(K^{\langle n\rangle}(\beta)\right.$ resp. $)$ and $K^{\langle n\rangle}(\alpha, \lambda)\left(K^{\langle n\rangle}(\beta, \lambda)\right.$ resp. $)$, respectively.
\end{abstract}

\section{Introduction and preliminaries}

We denote the class of analytic functions in the unit disk $\mathbb{D}=\{z \in \mathbb{C}$ : $|z|<1\}$ by $\mathcal{H}(\mathbb{D})$, and we think of $\mathcal{H}(\mathbb{D})$ as a topological vector space endowed with the topology of uniform convergence over compact subsets of $\mathbb{D}$. We let $K^{\langle n\rangle}(\alpha)\left(K^{\langle n\rangle}(\beta)\right.$ resp.), where $\alpha<1(\beta>1$ resp.), denote the set of analytic functions $f \in \mathcal{H}(\mathbb{D})$ which are generalized convex of order $\alpha$ in $\mathbb{D}$ (generalized $\beta$-starlike functions in $\mathbb{D}$ resp.). We recall that $f \in K^{\langle n\rangle}(\alpha)\left(f \in K^{\langle n\rangle}(\beta)\right.$ resp.) if and only if $f(0)=f^{\prime}(0)=\cdots=f^{(n-1)}(0)=f^{(n)}(0)-1=0$ and $\operatorname{Re}\left(P_{f}^{\langle n\rangle}(z)\right)>\alpha\left(\operatorname{Re}\left(P_{f}^{\langle n\rangle}(z)\right)<\beta\right.$ resp. $)$, where

$$
P_{f}^{\langle n\rangle}(z)=\frac{z f^{(n+1)}(z)}{f^{(n)}(z)}+1 \quad(z \in \mathbb{D})
$$

Received October 16, 2008.

2000 Mathematics Subject Classification. Primary 30C65, 30C45; Secondary 30C20.

Key words and phrases. Schwarz lemma, analytic function, univalent function, starlike function, generalized $\alpha$-convex domain, $\beta$-starlike function, region of variability, extreme point.

The research was partly supported by NSFs of China (No. 10571048) and of Hunan Province (No. 05JJ10001), and NCET (No. 04-0783). 
and "Re" denotes the real part.

Let $S_{n}^{*}$ denote the class of univalent starlike functions in $\mathbb{D}$ with $f(0)=$ $f^{\prime}(0)=\cdots=f^{(n-1)}(0)=f^{(n)}(0)-1=0$, where $f^{(n-1)}$ is univalent.

We recall that $K^{\langle n\rangle}(\beta) \subset S_{n}^{*}$ for $\beta=\frac{3}{2}$ (See [9]). It is well known that if $-\frac{1}{2} \leq \alpha<1$ and $f \in K^{\langle n\rangle}(\alpha)$, then $f^{(n-1)}$ is univalent (cf. [4]).

For $f \in K^{\langle n\rangle}(\alpha)\left(f \in K^{\langle n\rangle}(\beta)\right.$ resp.), we denote by $\log f^{(n)}$ the singlevalued branch of the logarithm of $f^{(n)}$ with $\log f^{(n)}(0)=0$. Using the well known Herglotz representation for analytic functions with positive real part in $\mathbb{D}$, we know that if $f \in K^{\langle n\rangle}(\alpha)$, then there exists a unique positive unit measure $\mu$ on $(-\pi, \pi]$ such that

$$
\frac{z f^{(n+1)}(z)}{f^{(n)}}+1=(1-\alpha) \int_{-\pi}^{\pi} \frac{1+z e^{-i t}}{1-z e^{-i t}} d \mu(t)+\alpha .
$$

Hence

$$
\log f^{(n)}(z)=2(1-\alpha) \int_{-\pi}^{\pi} \log \frac{1}{1-z e^{-i t}} d \mu(t) .
$$

It follows that for each fixed $z_{0} \in \mathbb{D}$ the region of variability

$$
V^{\langle n\rangle}\left(z_{0}, \alpha\right)=\left\{\log f^{(n)}\left(z_{0}\right): f \in K^{\langle n\rangle}(\alpha)\right\}
$$

coincides with the set

$$
\left\{-2(1-\alpha) \log (1-z):|z| \leq\left|z_{0}\right|\right\} .
$$

Similarly, if $f \in K^{\langle n\rangle}(\beta)$, then

$$
\frac{z f^{(n+1)}(z)}{f^{(n)}}+1=\beta-(\beta-1) \int_{-\pi}^{\pi} \frac{1+z e^{-i t}}{1-z e^{-i t}} d \mu(t) .
$$

Hence

$$
\log f^{(n)}(z)=2(\beta-1) \int_{-\pi}^{\pi} \log \left(1-z e^{-i t}\right) d \mu(t) .
$$

And so for each fixed $z_{0} \in \mathbb{D}$, the region of variability

$$
V^{\langle n\rangle}\left(z_{0}, \beta\right)=\left\{\log f^{(n)}\left(z_{0}\right): f \in K^{\langle n\rangle}(\beta)\right\}
$$

coincides with the set

$$
\left\{2(\beta-1) \log (1-z):|z| \leq\left|z_{0}\right|\right\} .
$$

Let $B_{0}$ be the class of analytic functions $\omega$ in $\mathbb{D}$ such that $|\omega(z)| \leq 1$ in $\mathbb{D}$ and $\omega(0)=0$. We see that if $f \in K^{\langle n\rangle}(\alpha)$, then

$$
\omega_{f}(z)=\frac{P_{f}^{\langle n\rangle}(z)-1}{P_{f}^{\langle n\rangle}(z)+1-2 \alpha} \in B_{0} .
$$


If $f \in K^{\langle n\rangle}(\beta)$, then

$$
\omega_{f}(z)=\frac{P_{f}^{\langle n\rangle}(z)-1}{P_{f}^{\langle n\rangle}(z)-2 \beta+1} \in B_{0}
$$

and conversely.

Schwarz Lemma implies that if $f \in K^{\langle n\rangle}(\alpha)$, then

$$
\left|f^{(n+1)}(0)\right|=\left|2(1-\alpha) \omega_{f}^{\prime}(0)\right| \leq 2(1-\alpha) .
$$

If $f \in K^{\langle n\rangle}(\beta)$, then

$$
\left|f^{(n+1)}(0)\right|=\left|2(1-\beta) \omega_{f}^{\prime}(0)\right| \leq 2(\beta-1) .
$$

For $\lambda \in \overline{\mathbb{D}}=\{z \in \mathbb{C}:|z| \leq 1\}$ and $z_{0} \in \mathbb{D}$, we introduce the following six notations.

$$
\begin{gathered}
K^{\langle n\rangle}(\alpha, \lambda)=\left\{f \in K^{\langle n\rangle}(\alpha): f^{(n+1)}(0)=2(1-\alpha) \lambda\right\}, \\
K^{\langle n\rangle}(\beta, \lambda)=\left\{f \in K^{\langle n\rangle}(\beta): f^{(n+1)}(0)=2(1-\beta) \lambda\right\}, \\
V^{\langle n\rangle}\left(z_{0}, \alpha, \lambda\right)=\left\{\log f^{(n)}\left(z_{0}\right): f \in K^{\langle n\rangle}(\alpha, \lambda)\right\}, \\
V^{\langle n\rangle}\left(z_{0}, \beta, \lambda\right)=\left\{\log f^{(n)}\left(z_{0}\right): f \in K^{\langle n\rangle}(\beta, \lambda)\right\}
\end{gathered}
$$

and

$$
\begin{aligned}
& \digamma_{\alpha}=\left\{P_{f}^{\langle n\rangle}(z): \operatorname{Re}\left(P_{f}^{\langle n\rangle}(z)\right)>\alpha, \text { where } \alpha<1\right\}, \\
& \digamma_{\beta}=\left\{P_{f}^{\langle n\rangle}(z): \operatorname{Re}\left(P_{f}^{\langle n\rangle}(z)\right)<\beta, \text { where } \beta>1\right\} .
\end{aligned}
$$

Herglotz formula shows that the extreme points of $\digamma_{\alpha}$ and $\digamma_{\beta}$ are precisely the functions

$$
f(z)=(1-\alpha) \frac{e^{i \theta}+z}{e^{i \theta}-z}+\alpha
$$

and

$$
f(z)=\beta-(\beta-1) \frac{e^{i \theta}+z}{e^{i \theta}-z}
$$

respectively.

Recently, the regions of variability for convex functions, functions of bounded derivative, and spirallike functions etc have been discussed by Ponnusamy, Vasudevarao, and Yanagihara. See $[10,11,14]$ for the details.

The main aim of this paper is to determine the sets $V^{\langle n\rangle}\left(z_{0}, \alpha\right), V^{\langle n\rangle}\left(z_{0}, \alpha\right.$, $\lambda), V^{\langle n\rangle}\left(z_{0}, \beta\right), V^{\langle n\rangle}\left(z_{0}, \beta, \lambda\right)$ and the extreme points of the families of analytic functions which satisfy $f(\mathbb{D}) \subset V^{\langle n\rangle}\left(z_{0}, \alpha\right)$ or $f(\mathbb{D}) \subset V^{\langle n\rangle}\left(z_{0}, \beta\right)$, respectively. Our main results are Theorems 2.2, 2.4, 2.6, 2.7, 2.8 and 2.9, where Theorems 2.2 and 2.4 are generalizations of the corresponding results in $[10,14]$. They will be proved in Section 3 . 


\section{Basic properties and main results}

For our presentation, we need some more preparation. Let $S^{*}=S_{1}^{*}$, i.e., the class of analytic functions $f$ in $\mathbb{D}$ with $f(0)=f^{\prime}(0)-1=0$ which map $\mathbb{D}$ conformally onto a starlike domain (with respect to the origin). Each function $f \in S^{*}$ is called starlike (univalent) in $\mathbb{D}$ and the function $f \in S^{*}$ is characterized by the analytic condition $\operatorname{Re}\left(z f^{\prime}(z) / f(z)\right)>0$ in $\mathbb{D}($ cf. $[2,3])$. For a positive integer $p$, let $\left(S^{*}\right)^{p}=\left\{f=f_{0}^{p}: f_{0} \in S^{*}\right\}$. Now we recall the following result which is from [3].

Lemma 2.1. Let $f$ be an analytic function in $\mathbb{D}$ with $f(z)=z^{p}+\cdots$. If for any $z \in \mathbb{D}, \operatorname{Re}\left(1+\frac{z f^{\prime \prime}(z)}{f^{\prime}(z)}\right)>0$, then $f \in\left(S^{*}\right)^{p}$.

We shall introduce some basic properties of $V^{\langle n\rangle}\left(z_{0}, \alpha\right), V^{\langle n\rangle}\left(z_{0}, \alpha, \lambda\right), V^{\langle n\rangle}$ $\left(z_{0}, \beta\right)$ and $V^{\langle n\rangle}\left(z_{0}, \beta, \lambda\right)$. Since the two sets $V^{\langle n\rangle}\left(z_{0}, \alpha\right), V^{\langle n\rangle}\left(z_{0}, \alpha, \lambda\right)$ and the two sets $V^{\langle n\rangle}\left(z_{0}, \beta\right), V^{\langle n\rangle}\left(z_{0}, \beta, \lambda\right)$ have the similar corresponding properties, in the following, we only list down some basic properties of $V^{\langle n\rangle}\left(z_{0}, \alpha\right)$ and $V^{\langle n\rangle}\left(z_{0}, \alpha, \lambda\right)$.

(I) $V^{\langle n\rangle}\left(z_{0}, \alpha, \lambda\right) \subset V^{\langle n\rangle}\left(z_{0}, \alpha\right)$.

(II) The sets $V^{\langle n\rangle}\left(z_{0}, \alpha\right)$ and $V^{\langle n\rangle}\left(z_{0}, \alpha, \lambda\right)$ are compact.

This statement follows from (1.2) and the fact that both $K^{\langle n\rangle}(\alpha)$ and $K^{\langle n\rangle}$ $(\alpha, \lambda)$ are closed in $\mathcal{H}(\mathbb{D})$.

(III) The sets $V^{\langle n\rangle}\left(z_{0}, \alpha\right)$ and $V^{\langle n\rangle}\left(z_{0}, \alpha, \lambda\right)$ are convex.

Indeed, if $f_{0}, f_{1} \in K^{\langle n\rangle}(\alpha)$ and $0 \leq t \leq 1$, then the function

$$
\begin{aligned}
f_{t}(z)=\int_{0}^{z} \int_{0}^{\zeta_{n}} \int_{0}^{\zeta_{n-1}} \cdots \int_{0}^{\zeta_{2}} \exp \{ & (1-t) \log f_{0}^{(n)}\left(\zeta_{1}\right) \\
& \left.+t \log f_{1}^{(n)}\left(\zeta_{1}\right)\right\} d \zeta_{1} d \zeta_{2} \cdots d \zeta_{n-1} d \zeta_{n}
\end{aligned}
$$

belongs to $K^{\langle n\rangle}(\alpha)$. Since $\log f_{t}^{(n)}\left(z_{0}\right)=(1-t) \log f_{0}^{(n)}\left(z_{0}\right)+t \log f_{1}^{(n)}\left(z_{0}\right)$, the convexity of $V^{\langle n\rangle}\left(z_{0}, \alpha\right)$ follows.

Similar reasoning shows that $V^{\langle n\rangle}\left(z_{0}, \alpha, \lambda\right)$ is also convex. is

(IV) If $|\lambda|=1$ or $z_{0}=0$, then $V^{\langle n\rangle}\left(z_{0}, \alpha, \lambda\right)$ consists of only one point which

$$
-2(1-\alpha) \log \left(1-\lambda z_{0}\right)
$$

If $|\lambda|<1$ and $z_{0} \neq 0$, then

$$
-2(1-\alpha) \log \left(1-\lambda z_{0}\right)
$$

is an interior point of the set $V^{\langle n\rangle}\left(z_{0}, \alpha, \lambda\right)$.

Indeed, if $|\lambda|=\left|\omega_{f}^{\prime}(0)\right|=1$, then it follows from Schwarz Lemma that $\omega_{f}(z)=\lambda z$, which implies that $P_{f}^{\langle n\rangle}(z)=\frac{1+(1-2 \alpha) \lambda z}{1-\lambda z}$ and $\log f^{(n)}(z)=-2(1-$ $\alpha) \log (1-\lambda z)$. This also trivially holds for the case $z_{0}=0$. 
For $\lambda \in \mathbb{D}$ and $a \in \overline{\mathbb{D}}$, let $\delta(z, \lambda)=\frac{z+\lambda}{1+\bar{\lambda} z}$ and

$$
\begin{aligned}
& F_{\langle n\rangle, a, \lambda}(z) \\
= & \int_{0}^{z}\left\{\int_{0}^{\zeta_{n+1}} \int_{0}^{\zeta_{n}} \cdots \int_{0}^{\zeta_{3}} \exp \left\{\int_{0}^{\zeta_{2}} \frac{2(1-\alpha) \delta\left(a \zeta_{1}, \lambda\right)}{1-\delta\left(a \zeta_{1}, \lambda\right) \zeta_{1}} d \zeta_{1}\right\} d \zeta_{2} \cdots d \zeta_{n}\right\} d \zeta_{n+1}
\end{aligned}
$$

for $z \in \mathbb{D}$.

Then, obviously,

$$
F_{\langle n\rangle, a, \lambda} \in K^{\langle n\rangle}(\alpha, \lambda)
$$

and

$$
\omega_{F_{\langle n\rangle, a, \lambda}}(z)=z \delta(a z, \lambda) .
$$

(V) The mapping $\mathbb{D} \ni a \mapsto \log F_{\langle n\rangle, a, \lambda}^{(n)}\left(z_{0}\right)$ is a non-constant analytic function of $a$ for any fixed $z_{0} \in \mathbb{D} \backslash\{0\}$ and $\lambda \in \mathbb{D}$.

Let

$$
\begin{aligned}
h(z) & =\frac{1}{(1-\alpha)\left(1-|\lambda|^{2}\right)} \frac{\partial}{\partial a}\left\{\log F_{\langle n\rangle, a, \lambda}^{(n)}(z)\right\}_{a=0} \\
& =2 \int_{0}^{z} \frac{\zeta}{(1-\lambda \zeta)^{2}} d \zeta=z^{2}+\cdots
\end{aligned}
$$

Then it is easy to see that $\frac{z h^{\prime \prime}(z)}{h^{\prime}(z)}+1=\frac{2}{1-z \lambda}$ and $\operatorname{Re}\left\{\frac{z h^{\prime \prime}(z)}{h^{\prime}(z)}+1\right\}>0$ for $z \in \mathbb{D}$.

By Lemma 2.1, there exists a function $h_{0} \in S^{*}$ with $h=h_{0}^{2}$. The univalence of $h_{0}$ and $h_{0}(0)=0$ imply that $h\left(z_{0}\right) \neq 0$ for $z_{0} \in \mathbb{D} \backslash\{0\}$. Consequently, the mapping $\mathbb{D} \ni a \mapsto \log F_{\langle n\rangle, a, \lambda}^{(n)}\left(z_{0}\right)$ is a non-constant analytic function of $a$. Property $(V)$ is proved.

It follows from Property $(\mathrm{V})$ that the mapping in $(\mathrm{V})$ is an open mapping. Hence $V^{\langle n\rangle}\left(z_{0}, \alpha, \lambda\right)$ contains the open set $\left\{\log F_{\langle n\rangle, a, \lambda}^{(n)}\left(z_{0}\right):|a|<1\right\}$. In particular,

$$
\log F_{\langle n\rangle, 0, \lambda}^{(n)}\left(z_{0}\right)=-2(1-\alpha) \log \left(1-z_{0} \lambda\right)
$$

is an interior point of the set $\left\{\log F_{\langle n\rangle, a, \lambda}^{(n)}\left(z_{0}\right): a \in \mathbb{D}\right\} \subset V^{\langle n\rangle}\left(z_{0}, \alpha, \lambda\right)$. Up to now, we finish the proof of Property (IV).

Finally, since $V^{\langle n\rangle}\left(z_{0}, \alpha, \lambda\right)$ is a compact convex subset of $\mathbb{C}$ and has nonempty interior, we see that the boundary $\partial V^{\langle n\rangle}\left(z_{0}, \alpha, \lambda\right)$ of $V^{\langle n\rangle}\left(z_{0}, \alpha, \lambda\right)$ is a Jordan curve and $V^{\langle n\rangle}\left(z_{0}, \alpha, \lambda\right)$ is the union of $\partial V^{\langle n\rangle}\left(z_{0}, \alpha, \lambda\right)$ and its inner domain.

(VI) $V^{\langle n\rangle}\left(z_{0} e^{i \theta}, \alpha, \lambda\right)=V^{\langle n\rangle}\left(z_{0}, \alpha, e^{i \theta} \lambda\right)$ for $\theta \in \mathbb{R}$.

This is a consequence of the fact that $e^{-i n \theta} f\left(e^{i \theta} z\right) \in K^{\langle n\rangle}\left(\alpha, \lambda e^{i \theta}\right)$ if and only if $f \in K^{\langle n\rangle}(\alpha, \lambda)$.

The following are our main results. 
Theorem 2.2. For $0 \leq \lambda<1$ and $z_{0} \in \mathbb{D} \backslash\{0\}$, the boundary $\partial V^{\langle n\rangle}\left(z_{0}, \alpha, \lambda\right)$ is the Jordan curve given by

$$
(-\pi, \pi] \ni \theta \mapsto \log F_{\langle n\rangle, e^{i \theta}, \lambda}^{(n)}\left(z_{0}\right)=\int_{0}^{z_{0}} \frac{2(1-\alpha) \delta\left(e^{i \theta} \zeta, \lambda\right)}{1-\delta\left(e^{i \theta} \zeta, \lambda\right) \zeta} d \zeta .
$$

If $\log f^{(n)}\left(z_{0}\right)=\log F_{\langle n\rangle, e^{i \theta}, \lambda}^{(n)}\left(z_{0}\right)$ for some $f \in K^{\langle n\rangle}(\alpha, \lambda)$ and $\theta \in(-\pi, \pi]$, then $f=F_{\langle n\rangle, e^{i \theta}, \lambda}$.

Remark 2.3. When $\alpha=0$, Theorem 2.2 coincides with Theorem 1.1 in [14], and when $\alpha=-\frac{1}{2}$, Theorem 2.2 coincides with Theorem 2.8 in [10].

Theorem 2.4. For $0 \leq \lambda<1$ and $z_{0} \in \mathbb{D} \backslash\{0\}$, the boundary $\partial V^{\langle n\rangle}\left(z_{0}, \beta, \lambda\right)$ is the Jordan curve given by

$$
(-\pi, \pi] \ni \theta \mapsto \log G_{\langle n\rangle, e^{i \theta}, \lambda}^{(n)}\left(z_{0}\right)=\int_{0}^{z_{0}} \frac{2(\beta-1) \delta\left(e^{i \theta} \zeta, \lambda\right)}{\delta\left(e^{i \theta} \zeta, \lambda\right) \zeta-1} d \zeta,
$$

where

$$
\begin{aligned}
& G_{\langle n\rangle, a, \lambda}(z) \\
= & \int_{0}^{z}\left\{\int_{0}^{\zeta_{n+1}} \int_{0}^{\zeta_{n}} \cdots \int_{0}^{\zeta_{3}} \exp \left\{\int_{0}^{\zeta_{2}} \frac{2(\beta-1) \delta\left(a \zeta_{1}, \lambda\right)}{\delta\left(a \zeta_{1}, \lambda\right) \zeta_{1}-1} d \zeta_{1}\right\} d \zeta_{2} \cdots d \zeta_{n}\right\} d \zeta_{n+1}
\end{aligned}
$$

for $z \in \mathbb{D}$.

If $\log f^{(n)}\left(z_{0}\right)=\log G_{\langle n\rangle, e^{i \theta}, \lambda}^{(n)}\left(z_{0}\right)$ for some $f \in K^{\langle n\rangle}(\beta, \lambda)$ and $\theta \in(-\pi, \pi]$, then $f=G_{\langle n\rangle, e^{i \theta}, \lambda}$.

Remark 2.5. When $\beta=\frac{3}{2}$, Theorem 2.4 coincides with Theorem 2.6 in [10].

As in [12], a proper domain $G$ of $\mathbb{C}$ is called a uniform domain provided there exists a constant $c(>0)$ such that each pair of points $z_{1}, z_{2} \in D$ can be joined by a rectifiable arc $\gamma \subset D$ for which

$$
l(\gamma) \leq c\left|z_{1}-z_{2}\right|
$$

and

$$
\min _{j=1,2} l\left(\gamma\left[z_{j}, z\right]\right) \leq c \operatorname{dist}(z, \partial D)
$$

for all $z \in \gamma$. Here $l(\gamma)$ denotes the Euclidean length of $\gamma, \gamma\left(z_{j}, z\right)$ the part of $\gamma$ between $z_{j}$ and $z$, and $\operatorname{dist}(z, \partial D)$ the Euclidean distance from $z$ to $\partial D$ which is the boundary of $D$.

The following two results easily follow from Properties (II), (III) as above and the well known fact that any bounded and convex proper domain of $\mathbb{C}$ is uniform (cf. [13]).

Theorem 2.6. For $z_{0} \in \mathbb{D} \backslash\{0\}$, the domains

$$
V^{\langle n\rangle}\left(z_{0}, \alpha\right) \backslash \partial V^{\langle n\rangle}\left(z_{0}, \alpha\right)
$$


and

are uniform.

$$
V^{\langle n\rangle}\left(z_{0}, \alpha, \lambda\right) \backslash \partial V^{\langle n\rangle}\left(z_{0}, \alpha, \lambda\right)
$$

Theorem 2.7. For $z_{0} \in \mathbb{D} \backslash\{0\}$, the domains

$$
V^{\langle n\rangle}\left(z_{0}, \beta\right) \backslash \partial V^{\langle n\rangle}\left(z_{0}, \beta\right)
$$

and

are uniform.

$$
V^{\langle n\rangle}\left(z_{0}, \beta, \lambda\right) \backslash \partial V^{\langle n\rangle}\left(z_{0}, \beta, \lambda\right)
$$

Theorem 2.8. Let $\digamma_{\alpha}^{*}$ denote the set of analytic functions in $\mathbb{D}$ so that $f(\mathbb{D}) \subset$ $V^{\langle n\rangle}\left(z_{0}, \alpha\right)$ and $f(0)=0$. Then a function $f$ in $\mathbb{D}$ is an extreme point of $\digamma_{\alpha}^{*}$ if and only if $f \in \digamma_{\alpha}^{*}$ and $\int_{0}^{2 \pi} \log \lambda(\theta) d \theta=-\infty$, where $\lambda(\theta)$ denotes the distance between $f\left(e^{i \theta}\right)\left(=\lim _{r \rightarrow 1} f\left(r e^{i \theta}\right)\right)$ and $\partial V^{\langle n\rangle}\left(z_{0}, \alpha\right)$.

Theorem 2.9. Let $\digamma_{\beta}^{*}$ denote the set of analytic functions in $\mathbb{D}$ so that $f(\mathbb{D}) \subset$ $V^{\langle n\rangle}\left(z_{0}, \beta\right)$ and $f(0)=0$. Then a function $f$ in $\mathbb{D}$ is an extreme point of $\digamma_{\beta}^{*}$ if and only if $f \in \digamma_{\beta}^{*}$ and $\int_{0}^{2 \pi} \log \lambda(\theta) d \theta=-\infty$, where $\lambda(\theta)$ denotes the distance between $f\left(e^{i \theta}\right)\left(=\lim _{r \rightarrow 1} f\left(r e^{i \theta}\right)\right)$ and $\partial V^{\langle n\rangle}\left(z_{0}, \beta\right)$.

As a simple application of Theorems 2.8 and 2.9 , we see that if

$$
f_{\alpha}(z)=2(1-\alpha) \log \frac{1}{1-z\left|z_{0}\right| e^{-i \theta}}
$$

and

$$
f_{\beta}(z)=2(\beta-1) \log \left(1-z\left|z_{0}\right| e^{-i \theta}\right),
$$

then $f_{\alpha}(z)$ and $f_{\beta}(z)$ are extreme points of $\digamma_{\alpha}^{*}$ and $\digamma_{\beta}^{*}$, respectively.

\section{Proofs of the main results}

It is enough to prove Theorems 2.2 and 2.8 since the proofs of Theorems 2.3 and 2.9 are similar.

We start with the following proposition which plays a key role in the proof of Theorem 2.2.

Proposition 3.1. For any $f \in K^{\langle n\rangle}(\alpha, \lambda)$, we know that for any $z \in \mathbb{D}$,

$$
\left|\frac{f^{(n+1)}(z)}{f^{(n)}(z)}-c(z, \lambda)\right| \leq r(z, \lambda)(z \in \mathbb{D}),
$$

where

$$
c(z, \lambda)=\frac{2(1-\alpha)\left\{\lambda\left(1-|z|^{2}\right)+\bar{z}\left(|z|^{2}-\lambda^{2}\right)\right\}}{\left(1-|z|^{2}\right)\left(1-\lambda(z+\bar{z})+|z|^{2}\right)}
$$

and

$$
r(z, \lambda)=\frac{2(1-\alpha)\left(1-\lambda^{2}\right)|z|}{\left(1-|z|^{2}\right)\left(1-\lambda(z+\bar{z})+|z|^{2}\right)} .
$$


For each $z \in \mathbb{D} \backslash\{0\}$, the equality sign in (3.1) holds if and only if $f=$ $F_{\langle n\rangle, e^{i \theta}, \lambda}$ for some $\theta \in \mathbb{R}$.

Proof. For any $f \in K^{\langle n\rangle}(\alpha, \lambda)$, let $\omega_{f} \in B_{0}$ be as in (1.3). Then $\omega_{f}^{\prime}(0)=\lambda$. It follows from Schwarz Lemma (see for example [2] or $[6,7,8]$ ) that

$$
\left|\frac{\frac{\omega_{f}(z)}{z}-\lambda}{1-\frac{\lambda \omega_{f}(z)}{z}}\right| \leq|z| \text {. }
$$

From (1.3), the inequality (3.2) is equivalent to

$$
\left|\frac{\frac{f^{(n+1)}(z)}{f^{(n)}(z)}-A(z, \lambda)}{\frac{f^{(n+1)}(z)}{f^{(n)}(z)}+B(z, \lambda)}\right| \leq|z||T(z, \lambda)|
$$

where

$$
A(z, \lambda)=\frac{2(1-\alpha) \lambda}{1-\lambda z}, B(z, \lambda)=\frac{2(1-\alpha)}{z-\lambda} \text { and } T(z, \lambda)=\frac{z-\lambda}{1-z \lambda} .
$$

We can see that the inequality (3.3) is equivalent to

$$
\begin{aligned}
& \left|\frac{f^{(n+1)}(z)}{f^{(n)}(z)}-\frac{\left[A(z, \lambda)+|z|^{2}|T(z, \lambda)|^{2} B(z, \lambda)\right]}{1-|z|^{2}|T(z, \lambda)|^{2}}\right| \\
\leq & \frac{|z||T(z, \lambda)||A(z, \lambda)+B(z, \lambda)|}{1-|z|^{2}|T(z, \lambda)|^{2}} .
\end{aligned}
$$

It follows from (3.4) that

and

$$
\begin{gathered}
1-|z|^{2}|T(z, \lambda)|^{2}=\frac{\left(1-|z|^{2}\right)\left(1+|z|^{2}-2 \lambda \operatorname{Re}(z)\right)}{|1-\lambda z|^{2}}, \\
A(z, \lambda)+B(z, \lambda)=\frac{2\left(1-\lambda^{2}\right)(1-\alpha)}{(1-\lambda z)(z-\lambda)}
\end{gathered}
$$

$$
A(z, \lambda)+|z|^{2}|T(z, \lambda)|^{2} B(z, \lambda)=\frac{2(1-\alpha)\left\{\lambda\left(1-|z|^{2}\right)+\bar{z}\left(|z|^{2}-\lambda^{2}\right)\right\}}{|1-\lambda z|^{2}} .
$$

Then we see that

and

$$
\frac{A(z, \lambda)+|z|^{2}|T(z, \lambda)|^{2} B(z, \lambda)}{1-|z|^{2}|T(z, \lambda)|^{2}}=c(z, \lambda)
$$

$$
\frac{|z||T(z, \lambda)||A(z, \lambda)+B(z, \lambda)|}{1-|z|^{2}|T(z, \lambda)|^{2}}=r(z, \lambda) .
$$

By (3.5), the inequality (3.1) follows.

It is easy to see that the equality sign occurs for any $z \in \mathbb{D} \backslash\{0\}$ in (3.1) if $f=F_{\langle n\rangle, e^{i \theta}, \lambda}$ for some $\theta \in \mathbb{R}$. Conversely, if the equality sign in (3.1) occurs for some $z \in \mathbb{D} \backslash\{0\}$, then the equality sign must hold in (3.2). Then Schwarz 
Lemma shows that there exists $\theta \in \mathbb{R}$ such that $\omega_{f}(z)=z \delta\left(e^{i \theta} z, \lambda\right)$ for all $z \in \mathbb{D}$. This implies that $f=F_{\langle n\rangle, e^{i \theta}, \lambda}$.

Corollary 3.2. Let $\gamma: t \longmapsto z(t)(0 \leq t \leq 1)$ be a $C^{1}$-curve in $\mathbb{D}$ with $z(0)=0$ and $z(1)=z_{0}$. Then we have

$V^{\langle n\rangle}\left(z_{0}, \alpha, \lambda\right) \subset \overline{\mathbb{D}}(C(\lambda, \gamma), R(\lambda, \gamma)) \triangleq\{w \in \mathbb{C}:|w-C(\lambda, \gamma)| \leq R(\lambda, \gamma)\}$,

where $C(\lambda, \gamma)=\int_{0}^{1} c(z(t), \lambda) z^{\prime}(t) d t$ and $R(\lambda, \gamma)=\int_{0}^{1} r(z(t), \lambda)\left|z^{\prime}(t)\right| d t$.

Proof. For any $f \in K^{\langle n\rangle}(\alpha, \lambda)$, it follows from Proposition 3.1 that

$$
\begin{aligned}
\left|\log f^{(n)}\left(z_{0}\right)-C(\lambda, \gamma)\right| & =\left|\int_{0}^{1}\left\{\frac{f^{(n+1)}(z(t))}{f^{(n)}(z(t))}-c(z(t), \lambda)\right\} z^{\prime}(t) d t\right| \\
& \leq \int_{0}^{1}\left|\frac{f^{(n+1)}(z(t))}{f^{(n)}(z(t))}-c(z(t), \lambda)\right|\left|z^{\prime}(t)\right| d t \\
& \leq \int_{0}^{1} r(z(t), \lambda)\left|z^{\prime}(t)\right| d t=R(\lambda, \gamma) .
\end{aligned}
$$

The proof is complete.

We recall the following result from [10], which is useful for the proof of Proposition 3.4.

Lemma 3.3. For $\theta \in \mathbb{R}$ and $\lambda \in[0,1]$, the function

$$
G(z)=\int_{0}^{z} \frac{e^{i \theta} \zeta}{\left\{1+\lambda\left(e^{i \theta}-1\right) \zeta-e^{i \theta} \zeta^{2}\right\}^{2}} d \zeta(z \in \mathbb{D})
$$

has a double zero at the origin and no zeros elsewhere in $\mathbb{D}$.

Furthermore, there exists a starlike univalent function $G_{0}$ in $\mathbb{D}$ such that $G=2^{-1} e^{i \theta} G_{0}^{2}$ and $G_{0}(0)=G_{0}^{\prime}(0)-1=0$.

Proposition 3.4. Let $z_{0} \in \mathbb{D} \backslash\{0\}$. Then for any $\theta \in(-\pi, \pi]$, we have $\log F_{\langle n\rangle, e^{i \theta, \lambda}}^{(n)}\left(z_{0}\right) \in \partial V^{\langle n\rangle}\left(z_{0}, \alpha, \lambda\right)$.

Furthermore, if $\log f^{(n)}\left(z_{0}\right)=\log F_{\langle n\rangle, e^{i \theta, \lambda}}^{(n)}\left(z_{0}\right)$ for some $f \in K^{\langle n\rangle}(\alpha, \lambda)$ and $\theta \in(-\pi, \pi]$, then $f=F_{\langle n\rangle, e^{i \theta}, \lambda}$.

Proof. From (2.2), we have

$$
\begin{aligned}
\frac{F_{\langle n\rangle, a, \lambda}^{(n+1)}(z)}{F_{\langle n\rangle, a, \lambda}^{(n)}(z)} & =\frac{2(1-\alpha) \delta(a z, \lambda)}{1-\delta(a z, \lambda)} \\
& =\frac{2(1-\alpha)(\lambda+a z)}{1+\lambda(a-1) z-a z^{2}} .
\end{aligned}
$$

It follows from (3.4) that

$$
\frac{F_{\langle n\rangle, a, \lambda}^{(n+1)}(z)}{F_{\langle n\rangle, a, \lambda}^{(n)}(z)}-A(z, \lambda)=\frac{\left(1-\lambda^{2}\right) a z 2(1-\alpha)}{(1-z \lambda)\left(1+\lambda(a-1) z-a z^{2}\right)}
$$


and

$$
\frac{F_{\langle n\rangle, a, \lambda}^{(n+1)}(z)}{F_{\langle n\rangle, a, \lambda}^{(n)}(z)}+B(z, \lambda)=\frac{2\left(1-\lambda^{2}\right)(1-\alpha)}{(z-\lambda)\left(1+\lambda(a-1) z-a z^{2}\right)} .
$$

Hence we obtain that

$$
\begin{aligned}
\frac{F_{\langle n\rangle, a, \lambda}^{(n+1)}(z)}{F_{\langle n\rangle, a, \lambda}^{(n)}(z)}-c(z, \lambda)= & \frac{F_{\langle n\rangle, a, \lambda}^{(n+1)}(z)}{F_{\langle n\rangle, a, \lambda}^{(n)}(z)}-\frac{A(z, \lambda)+|z|^{2}|T(z, \lambda)|^{2} B(z, \lambda)}{1-|z|^{2}|T(z, \lambda)|^{2}} \\
= & \frac{1}{1-|z|^{2}|T(z, \lambda)|^{2}}\left\{\left(\frac{F_{\langle n\rangle, a, \lambda}^{(n+1)}(z)}{F_{\langle n\rangle, a, \lambda}^{(n)}(z)}-A(z, \lambda)\right)\right. \\
& \left.-|z|^{2}|T(z, \lambda)|^{2}\left(\frac{F_{\langle n\rangle, a, \lambda}^{(n+1)}(z)}{F_{\langle n\rangle, a, \lambda}^{(n)}(z)}+B(z, \lambda)\right)\right\} \\
= & \frac{2(1-\alpha)\left(1-\lambda^{2}\right)\left\{a z(1-\lambda \bar{z})-|z|^{2}(\bar{z}-\lambda)\right\}}{\left(1-|z|^{2}\right)\left\{1-2 \lambda \operatorname{Re}(z)+|z|^{2}\right\}\left\{1+\lambda(a-1) z-a z^{2}\right\}} .
\end{aligned}
$$

Substituting $a$ by $e^{i \theta}$ in the above equalities we see that

$$
\begin{aligned}
& \frac{F_{\langle n\rangle, e^{i \theta}, \lambda}^{(n+1)}(z)}{F_{\langle n\rangle, e^{i \theta}, \lambda}^{(n)}(z)}-c(z, \lambda) \\
= & r(z, \lambda) \frac{\left|1+\lambda\left(e^{i \theta}-1\right) z-e^{i \theta} z^{2}\right|^{2}}{|z|} \frac{e^{i \theta} z}{\left[1+\lambda\left(e^{i \theta}-1\right) z-e^{i \theta} z^{2}\right]^{2}} .
\end{aligned}
$$

It follows from Lemma 3.3 that

$$
\frac{F_{\langle n\rangle, e^{i \theta}, \lambda}^{(n+1)}(z)}{F_{\langle n\rangle, e^{i \theta}, \lambda}^{(n)}(z)}-c(z, \lambda)=r(z, \lambda) \frac{G^{\prime}(z)}{\left|G^{\prime}(z)\right|} .
$$

Since the function $G_{0}$ is starlike, we see that for any $z_{0} \in \mathbb{D} \backslash\{0\}$, the linear segment joining 0 and $G_{0}\left(z_{0}\right)$ entirely lies in $G_{0}(\mathbb{D})$. Now we define $\gamma_{0}$ as follows.

$$
\gamma_{0}: t \longmapsto z(t)=G_{0}^{-1}\left(t G_{0}\left(z_{0}\right)\right)(0 \leq t \leq 1)
$$

Since

$$
G(z(t))=2^{-1} e^{i \theta} G_{0}(z(t))^{2}=2^{-1} e^{i \theta}\left(t G_{0}\left(z_{0}\right)\right)^{2}=t^{2} G\left(z_{0}\right),
$$

we have

$$
G^{\prime}(z(t)) z^{\prime}(t)=2 t G\left(z_{0}\right)(t \in[0,1])
$$


By (3.6) and (3.8), we have that

$(3.9)$

$$
\begin{aligned}
\log F_{\langle n\rangle, e^{i \theta, \lambda}}^{(n)}\left(z_{0}\right)-C\left(\lambda, \gamma_{0}\right) & =\int_{0}^{1}\left\{\frac{F_{\langle n\rangle, e^{i \theta}, \lambda}^{(n+1)}(z(t))}{F_{\langle n\rangle, e^{i \theta}, \lambda}^{(n)}(z(t))}-c(z(t), \lambda)\right\} z^{\prime}(t) d t \\
& =\int_{0}^{1} r(z(t), \lambda) \frac{G^{\prime}(z(t)) z^{\prime}(t)}{\left|G^{\prime}(z(t)) z^{\prime}(t)\right|}\left|z^{\prime}(t)\right| d t \\
& =\frac{G\left(z_{0}\right)}{\left|G\left(z_{0}\right)\right|} \int_{0}^{1} r(z(t), \lambda)\left|z^{\prime}(t)\right| d t \\
& =\frac{G\left(z_{0}\right)}{\left|G\left(z_{0}\right)\right|} R\left(\lambda, \gamma_{0}\right) .
\end{aligned}
$$

It yields that

$$
\log F_{\langle n\rangle, e^{i \theta}, \lambda}^{(n)}\left(z_{0}\right) \in \partial \overline{\mathbb{D}}\left(C\left(\lambda, \gamma_{0}\right), R\left(\lambda, \gamma_{0}\right)\right) .
$$

Also from Corollary 3.2, we know that

$$
\log F_{\langle n\rangle, e^{i \theta}, \lambda}^{(n)}\left(z_{0}\right) \in V^{\langle n\rangle}\left(z_{0}, \alpha, \lambda\right) \subset \overline{\mathbb{D}}\left(C\left(\lambda, \gamma_{0}\right), R\left(\lambda, \gamma_{0}\right)\right) .
$$

Hence we can conclude that

$$
\log F_{\langle n\rangle, e^{i \theta, \lambda}}^{(n)}\left(z_{0}\right) \in \partial V^{\langle n\rangle}\left(z_{0}, \alpha, \lambda\right) .
$$

Assume that there is some $f \in K^{\langle n\rangle}(\alpha, \lambda)$ and $\theta \in(-\pi, \pi]$ such that

$$
\log f^{(n)}\left(z_{0}\right)=\log F_{\langle n\rangle, e^{i \theta}, \lambda}^{(n)}\left(z_{0}\right) .
$$

Let

$$
h(t)=\frac{\overline{G\left(z_{0}\right)}}{\left|G\left(z_{0}\right)\right|}\left\{\frac{f^{(n+1)}(z(t))}{f^{(n)}(z(t))}-c(z(t), \lambda)\right\} z^{\prime}(t),
$$

where $z(t) \in \gamma_{0}$ which is given by (3.7). Then $h(t)$ is a continuous function in $[0,1]$ and (3.6) yields that

$$
|h(t)| \leq r(z(t), \lambda)\left|z^{\prime}(t)\right| .
$$

Furthermore, (3.9) and (3.10) yield that

$$
\begin{aligned}
\int_{0}^{1} \operatorname{Re}(h(t)) d t & =\int_{0}^{1} \operatorname{Re}\left\{\frac{\overline{G\left(z_{0}\right)}}{\left|G\left(z_{0}\right)\right|}\left[\frac{f^{(n+1)}(z(t))}{f^{(n)}(z(t))}-c(z(t), \lambda)\right] z^{\prime}(t)\right\} d t \\
& =\operatorname{Re}\left\{\frac{\overline{G\left(z_{0}\right)}}{\left|G\left(z_{0}\right)\right|}\left\{\log f^{(n)}\left(z_{0}\right)-C\left(\lambda, \gamma_{0}\right)\right\}\right\} \\
& =\operatorname{Re}\left\{\frac{\overline{G\left(z_{0}\right)}}{\left|G\left(z_{0}\right)\right|}\left\{\log F_{\langle n\rangle, e^{i \theta}, \lambda}^{(n)}\left(z_{0}\right)-C\left(\lambda, \gamma_{0}\right)\right\}\right\} \\
& =\int_{0}^{1} r(z(t), \lambda)\left|z^{\prime}(t)\right| d t .
\end{aligned}
$$


Thus we have $h(t)=r(z(t), \lambda)\left|z^{\prime}(t)\right|$ for all $t \in[0,1]$. It follows from (3.6) and (3.8) that for any $z \in \gamma_{0}$,

$$
\frac{f^{(n+1)}(z)}{f^{(n)}(z)}=\frac{F_{\langle n\rangle, e^{i \theta}, \lambda}^{(n+1)}(z)}{F_{\langle n\rangle, e^{i \theta}, \lambda}^{(n)}(z)} .
$$

Hence for any $z \in \mathbb{D}$,

$$
\frac{f^{(n+1)}(z)}{f^{(n)}(z)}=\frac{F_{\langle n\rangle, e^{i \theta, \lambda}}^{(n+1)}(z)}{F_{\langle n\rangle, e^{i \theta, \lambda}}^{(n)}(z)} .
$$

This implies that $f=F_{\langle n\rangle, e^{i \theta}, \lambda}$ in $\mathbb{D}$.

Proof of Theorem 2.2. At first, we prove that the closed curve

$$
(-\pi, \pi] \ni \theta \mapsto \log F_{\langle n\rangle, e^{i \theta, \lambda}}^{(n)}\left(z_{0}\right)
$$

is simple.

Suppose not. Then there are $\theta_{1}, \theta_{2} \in(-\pi, \pi]$ with $\theta_{1} \neq \theta_{2}$ such that

$$
\log F_{\langle n\rangle, e^{i \theta_{1}, \lambda}}^{(n)}\left(z_{0}\right)=\log F_{\langle n\rangle, e^{i \theta_{2}, \lambda}}^{(n)}\left(z_{0}\right) .
$$

By Proposition 3.4, we have $\log F_{\langle n\rangle, e^{i \theta_{1}, \lambda}}^{(n)}=\log F_{\langle n\rangle, e^{i \theta_{2}, \lambda}}^{(n)}$. It follows from (2.3) that $\theta_{1}=\theta_{2}$. This contradiction shows that the curve must be simple.

Since $V^{\langle n\rangle}\left(z_{0}, \alpha, \lambda\right)$ is a compact convex subset of $\mathbb{C}$ and has nonempty interior, we see that the boundary $\partial V^{\langle n\rangle}\left(z_{0}, \alpha, \lambda\right)$ is a simple closed curve. It follows from Proposition 3.4 that the curve $(-\pi, \pi] \ni \theta \mapsto \log F_{\langle n\rangle, e^{i \theta, \lambda}}^{(n)}\left(z_{0}\right)$ is a subcurve of $\partial V^{\langle n\rangle}\left(z_{0}, \alpha, \lambda\right)$.

The fact that a simple closed curve cannot contain any simple closed curve other than itself yields that $\partial V^{\langle n\rangle}\left(z_{0}, \alpha, \lambda\right)$ is given by

$$
(-\pi, \pi] \ni \theta \mapsto \log F_{\langle n\rangle, e^{i \theta}, \lambda}^{(n)}\left(z_{0}\right) .
$$

The proof of Theorem 2.8. From (1.2), we know that $V^{\langle n\rangle}\left(z_{0}, \alpha\right)$ is a bounded convex domain and has smooth boundary with positive curvature. Hence, Theorem 2.8 follows from Theorem 3 in [1].

Acknowledgement. The authors heartily thank professor S. Ponnusamy for his help for this paper and the authors also would like to thank the referee for the useful suggestions.

\section{References}

[1] Y. Abu-Muhanna and T. H. MacGregor, Extreme points of families of analytic functions subordinate to convex mappings, Math. Z. 176 (1981), no. 4, 511-519.

[2] P. L. Duren, Univalent Function, Grundlehren der mathematicchen Wissenschaften 259, New York, Berlin, Heidelberg, Tokyo, Spring-Verlag, 1983.

[3] A. W. Goodman, Univalent Functions, Vols. I and II, Mariner Publishing Co., Tampa, Florida, 1983. 
[4] D. J. Hallenbeck, Extreme points of classes of functions defined by subordination, Proc. Amer. Math. Soc. 46 (1974), 59-64.

[5] D. J. Hallenbeck and A. E. Livingston, Applications of extreme point theory to classes of multivalent functions, Trans. Amer. Math. Soc. 221 (1976), no. 2, 339-359.

[6] Ch. Pommerenke, Boundary Behaviour of Conform Maps, Springer-Verlag, Berlin, 1992.

[7] S. Ponnusamy, Foundations of Functional Analysis, Alpha Science International Ltd., Pangbourne, 2002.

[8] S. Ponnusamy and H. Silverman, Complex Variables with Applications, Birkhäuser, Boston, 2006.

[9] S. Ponnusamy and V. Singh, Univalence of certain integral transforms, Glas. Mat. Ser. III 31(51) (1996), no. 2, 253-261.

[10] S. Ponnusamy and A. Vasudevarao, Region of variability of two subclasses of univalent functions, J. Math. Anal. Appl. 332 (2007), no. 2, 1323-1334.

[11] S. Ponnusamy, A. Vasudevarao, and H. Yanagihara, Region of variability of univalent functions $f(z)$ for which $z f^{\prime}(z)$ is spirallike, Houston J. Math. 34 (2008), no. 4, 10371048.

[12] J. Väisälä, Uniform domains, Tohoku Math. J. (2) 40 (1988), no. 1, 101-118.

[13] X. Wang, M. Huang, and Y. Chu, Bounded and convex domains in $\mathbf{R}^{n}$, Acta Math. Sinica (Chin. Ser.) 50 (2007), no. 3, 481-484.

[14] H. Yanagihara, Regions of variability for functions of bounded derivatives, Kodai Math. J. 28 (2005), no. 2, 452-462.

Shaolin Chen

Department of Mathematics

HUNAN NORMAL UNIVERSITY

Changsha, Hunan 410081, P. R. China

E-mail address: shlchen1982@yahoo.com.cn

Aiwu Huang

Department of Mathematics

Hunan University of Chinese Medicine

Changsha, Hunan 410081, P. R. China

E-mail address: hezuoyun@sina.com 